\title{
EL IMPACTO DE LAS ESTRATEGIAS EN EL MARKETING MIX DE LA MIPYME EN LA ZONA DE LA PURÍSIMA EN EL MUNICIPIO DE AGUASCALIENTES, MÉXICO
}

The impact of strategies in the marketing mix of MSMEs in the Purisima Aguascalientes, Mexico

\author{
Gabriela Ortiz Delgadillo \\ Maestría en Mercadotecnia. Universidad Politécnica de Aguascalientes, Aguascalientes - México, \\ gabriela.ortiz@upa.edu.mx \\ Roussell Li-erth Toraya Pedroza \\ Maestría en Gestión Pública. Universidad Tecnológica del Norte de Aguascalientes, Aguascalientes - México, \\ roussell.toraya@utna.edu.mx

\section{Oscar Femat Esparza} \\ Maestría en Administración. Universidad Interamericana para el Desarrollo, Aguascalientes - México, \\ ofemat@unid.mx
}

\section{Cómo citar / How to cite}

Ortiz, G., Toraya, R. y Femat, O. (2015). El impacto de las estrategias de marketing de la Mipyme en la zona de la Purísima en el municipio de Aguascalientes. Revista CEA, 1(2), 49-61.

Recibido: 3 de mayo de 2015

Aceptado: 25 de junio de 2015

\begin{abstract}
Resumen
La presente investigación fue realizada con el objetivo de conocer el impacto de las estrategias en el Marketing mix de las Mipymes en la zona de La Purísima del municipio de Aguascalientes. Se realizó un estudio cuantitativo, utilizando un modelo descriptivocorrelacional, de corte trasversal, aplicado el instrumento a una muestra de 133 propietarios de las microempresas del sector comercial en La Purísima. Para el análisis de los datos, se apoyó en la técnica estadística de regresión lineal con apoyo del software SPSS versión 20, en donde los resultados muestran ser representativos, es decir, las estrategias exploradoras y defensivas impactan más en el Marketing mix en comparación de las estrategias analizadoras y reactivas. Es de suma importancia que los dueños de estas empresas decidan hacer uso de la investigación de mercados, esto les permitiría conocer qué está demandando el mercado y qué técnicas especializadas de marketing pueden utilizar para que el Marketing mix evolucione y se acondicione a los cambios del mercado.
\end{abstract}

Palabras clave: estrategias, Marketing mix y Mipyme.

\begin{abstract}
This research was conducted in order to ascertain the impact of the strategies in the Marketing mix of MSMEs in the area of the municipality of Aguascalientes Purisima. A quantitative study was conducted using a descriptive correlational, crosssectional model, applying the instrument to a sample of 133 owners of microenterprises in the business sector in La Purísima. For the data analysis, the statistical technique of linear regression with the support of SPSS version 20 was used. The results showed to be meaningful, i.e., exploratory and defensive strategies have a greater impact on the Marketing mix compared to the analyzer and reactive strategies. It is a crucial aspect that the owners of these companies decide to use the market research, since this would allow them to know what the market is demanding and what specialized marketing techniques can be used for the Marketing mix to evolve and reflects the market changes.
\end{abstract}

Keywords: Marketing mix, Strategies, and MSMEs business sector. 


\section{INTRODUCCIÓN}

Tomando en cuenta el nuevo paradigma de desarrollo y competitividad, basado en la nueva economía y que se caracteriza por una creciente globalización de intercambios comerciales, sumado a la aplicación de más sofisticados y complejos sistemas productivos, ha transformado el entorno competitivo global, por lo que las empresas están encontrando grandes dificultades para mantener sus ventajas competitivas. Para una Mipyme es aún más difícil ya que estas empresas tienen serias dificultades para sobrevivir y desarrollarse en un mercado competitivo, ya que actualmente es notable el interés por conocer las estrategias que mejoran la competitividad, permitiendo con ello alcanzar mejores resultados (Dávila y Rojo, 2010).

Para Aragón et. al (2010) las Mipymes tienen diferentes objetivos estratégicos, como son la búsqueda del máximo beneficio, la mejora de la productividad y el aumento del éxito competitivo; es por eso que actualmente es reconocida la creciente importancia de la pequeña y mediana empresa, en especial en los países en vía de desarrollo, por su aporte al empleo y al bienestar económico (Cardozo, 2012), ya que estas contribuyen con la generación de riqueza y el desarrollo equilibrado de las actividades económicas de un país.

Como consecuencia de la importancia que tienen las Mipyme en el contexto económico y social, estas se han convertido en foco de interés de los gobiernos de diversos países, ya que en gran parte del mundo se están estableciendo programas de apoyo e incentivos para la creación de pequeñas y medianas empresas debido al impacto económico y social que generan en el contexto nacional e internacional; por eso es prioritario realizar estudios que permitan identificar aquellos puntos débiles que pueden comprometer su continuidad o su funcionamiento de manera eficiente y eficaz (González, 2010).

Cuando se habla de factores sobre los cuales las
Mipymes basan su éxito se tiene que tomar en cuenta lo siguiente: la posición tecnológica, la innovación, la calidad del producto o servicio y la capacitación del personal (Rubio y Aragón, 2009). Indudablemente, uno de los factores más importantes y menos tomado en cuenta por parte de los empresarios de esta clasificación de empresas es el Marketing mix, el cual se compone de elementos que integran las estrategias, identificados y comúnmente conocidos como las 4P: producto, precio, plaza y promoción, de las cuales se busca la integración y combinación, en donde la información recolectada y analizada sobre cada una de ellas, tiene, no solo implicaciones para la toma de decisiones sobre la variable en cuestión sino también para el conjunto restante de estas (González, 2014).

En el mundo existen gran variedad de conceptos para considerar y definir a las micro, pequeñas y medianas empresas, ya que no hay una clasificación que englobe todas las características que tienen que cumplir para ser clasificadas en alguno de estos términos, y mucho depende de las necesidades propias de cada país o de los objetivos que se persigan. Sin embargo, en la Tabla 1 se muestra la estratificación de las microempresas por número de trabajadores, considerado desde el punto de vista del Diario Oficial de la Federación en México.

Cada país define a las Mipymes utilizando sus propios índices o características, pero no existe un índice único que caracterice la dimensión de la empresa de manera adecuada. Algunas de las variables tomadas en cuenta para establecer el tamaño o magnitud de las empresas son las siguientes: el número de trabajadores que emplean, el tipo de producto, el tamaño de mercado, la inversión en bienes de producción por persona ocupada, el volumen de producción o de ventas, el valor de producción o de ventas, el trabajo personal de socios o directores, la separación de funciones básicas de producción, personal, financieras y ventas dentro de la empresa, la ubicación o localización y el nivel de tecnología de producción (Zevallos, 2003). 
Tabla 1. Estratificación por Número de Trabajadores

Table 1. Stratification by Number of Workers

\begin{tabular}{ccccc} 
TAMAÑO & SECTOR & $\begin{array}{c}\text { RANGO DE NÚMEROS DE } \\
\text { TRABAJADORES }\end{array}$ & $\begin{array}{c}\text { RANGO DE MONTO DE VENTAS } \\
\text { ANUALES (MDP) }\end{array}$ & $\begin{array}{c}\text { TOPE MÁXIMO } \\
\text { COMBINADO }\end{array}$ \\
Micro & Todos & Hasta 10 & Hasta $\$ 4$ & 4.6 \\
\hline
\end{tabular}

*Tope Máximo Combinado=(Trabajadores) X 10\% + (Ventas Anuales) X 90\%

Fuente: Ley para el Desarrollo de la Competitividad de la Micro, Pequeña y Mediana Empresa del año 2002 DOF. 14-03-2014.

La presente investigación tiene como objetivo conocer el impacto de las estrategias en el Marketing mix de las Mipymes en la zona de la Purísima del municipio de Aguascalientes, con ello los microempresarios o propietarios podrán contar con información adecuada que les permita incidir para la toma de decisiones en términos de Marketing y desarrollo de estrategias empresariales. Para ello, es importante establecer el cuestionamiento de si las estrategias influyen de manera positiva en el Marketing mix que desarrollan dichas empresas, y a su vez, cuestionar cuál estrategia genera mayor impacto.

\section{MARCO TEÓRICO}

\section{Marketing mix}

La importancia que han adquirido variables como las estrategias de mercadotecnia y la innovación, se manifiesta en que ya no basta con producir un producto de calidad o a bajo costo, sino que la empresa debe ser capaz de hacer entender al cliente que está recibiendo el mejor producto, lo mejor de la empresa, donde la mezcla de mercadotecnia juega un papel muy importante, dado que una combinación única de estos elementos en una determinada industria permite a las empresas competir más eficazmente, garantizando la rentabilidad y la sostenibilidad de la misma (Castañeda, 2009; Uribe, 2010).

La mezcla de mercadotecnia es uno de los elementos del plan de mercadotecnia, que se puede definir como el conjunto de estrategias, técnicas y factores que ayudan a influir en el segmento del mercado donde se trabaja con los elementos que en su conjunto forman $y$ distinguen un producto (Montaño, 2005). A su vez, el Marketing mix es un conjunto de instrumentos tácticos controlables que la empresa combina para generar la respuesta deseada en el mercado objetivo, incluye todas las medidas tácticas que puede tomar una empresa para influir en la demanda de sus productos (Kotler y Armstrong, 2004). Se lleva a cabo cuando se elabora un producto que cumpla con las necesidades y deseos de los consumidores examinados con detenimiento y se ofrece a un precio determinado, se pone a disponibilidad en lugares o canales de distribución particulares y se lleva a cabo un plan de promoción o comunicación que genere interés y facilite los procesos de intercambio y el desarrollo de relaciones (Blech y Blech, 2004).

Alcántara (2012) menciona que el conjunto de variables que atiende la mercadotecnia para lograr los objetivos de la empresa y desarrollar estrategias de marketing se conocen como Marketing mix o mezcla de mercadotecnia; esta mezcla se conforma de 4 variables:

- Producto o servicio: es todo aquello tangible o intangible que ofrece un mercado para su adquisición, uso o consumo y que satisfacen una necesidad o deseo. Los aspectos que conforman esta variable son la formulación y presentación del producto, la marca, el empaque, etiqueta y envase; así como la 
administración del ciclo de vida del producto.

- Precio: es el monto monetario de la transacción. Entre los aspectos se encuentra formas de pago, tipos de crédito, descuentos. Los objetivos de marketing, la estrategia de la mezcla de mercadotecnia, los costos y los factores de organización. Representan los elementos a considerar por la empresa para el establecimiento del precio.

- Plaza: consiste en definir en dónde se comercializará el producto o servicio que se ofrece. Se considera el manejo efectivo de canales de distribución.

- Promoción: es la manera de comunicar, informar y persuadir al cliente y los interesados sobre la empresa, sus productos y ofertas. En este aspecto se manejan la promoción de ventas, la fuerza de ventas, la publicidad y las relaciones públicas, así como la comunicación interactiva. Estas variables se visualizan a través de las estrategias de mercadotecnia, que permiten alcanzar las metas y objetivos que pretende alcanzar la empresa.

Actualmente las pequeñas y medianas empresas, a través de la gestión de la mercadotecnia, pueden expandir su mercado satisfaciendo las necesidades de los clientes, haciendo eficiente el logro de los objetivos mediante la dirección de un personal apto e implementando técnicas que permitan conocer mejor el mercado, la competencia y el entorno. La implementación de una correcta mezcla de mercadotecnia depende en su mayoría de las características de cada empresa, así como de sus productos o servicios a ofrecer, ya que estos van relacionados con el correcto funcionamiento tanto de la mezcla como de la competitividad (Uribe, 2008).

Sin embargo, se debe tener presente que cada una de las 4P podría ser desglosada en numerosos subcomponentes, ya que la mezcla de marketing no se limita a solo $4 \mathrm{P}$, sino que puede consistir en otros factores que también ejercen influencia, significando que es necesario, además, identificar los sub componentes significativos que subyacen en la estrategia de mercadotecnia de la compañía (Monteiro, 2015; Islas, 2010).

Con base en lo anterior y a los cambios constantes es que la mezcla de mercadotecnia ha pasado de estar centrada en el producto a tener como enfoque al cliente, debido sobre todo, a la explosión de Internet y de las redes sociales, por lo que ahora es más viable una mercadotecnia basada en los consumidores y no en el producto; partiendo de esto se han introducido un nuevo conjunto de principios que han desplazado a las tradicionales variables del Marketing mix, dando paso a las 4C: cliente, costo, conveniencia y comunicación. En la Fig. 1 se muestra una comparación de las $4 \mathrm{P}$ con las $4 \mathrm{C}$ del consumidor.

Respecto al consumidor, se busca analizar las necesidades del cliente y en base a ello diseñar y producir el producto o servicio a ofrecer, así como las acciones de marketing para la presentación del producto. El costo es la relación entre el costo-beneficio, analizado desde el punto de vista del consumidor; el costo del producto es solo un aspecto del precio, lo que este factor involucra es considerar el beneficio que obtiene el consumidor más allá de cubrirle una necesidad, así como el costo en que incurre para poder acceder al producto y la conveniencia de la transacción, no modifica el costo real del producto o servicio pero sí influye en el costo final.

La conveniencia son las diferentes formas de comercialización, tales como las ventas por Internet y por catálogo, ofertas de grandes centros comerciales, la concentración de productos, el uso de tarjetas de crédito y los hábitos de compra, lo cual se presenta como un factor alterno a la plaza y a los canales tradicionales de distribución, permitiendo un mejor acceso del consumidor al producto. La comunicación se centra principalmente en establecer una comunicación de doble vía entre la empresa y el consumidor, al cual se considera como una persona con necesidades y deseos y no 
un simple cliente, esto permite establecer un vínculo de comunicación con el cliente más provechoso y la obtención de mucha más información sobre las personas, sus expectativas y necesidades y las motivaciones que influyen en su decisión de compra; lo que permite detectar nuevas necesidades y orientar la actividad empresarial con los productos que el mercado quiere. Aun así, la promoción y publicidad siguen usándose pero la comunicación entre los dos entes toma nueva importancia (McClean, 2012).

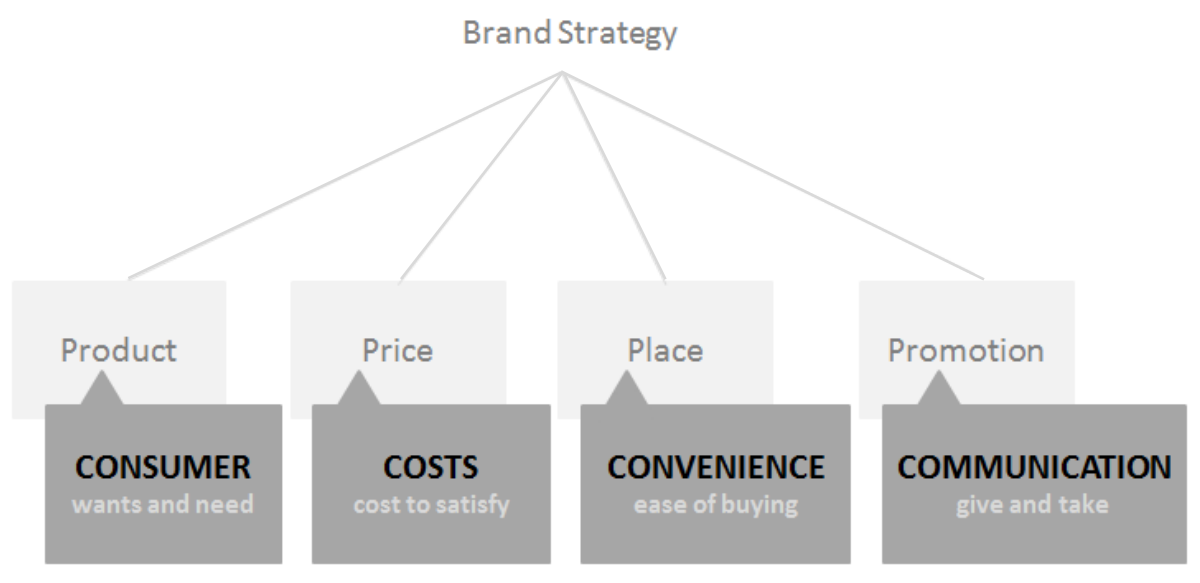

Figura 1. Las $4 \mathrm{P}$ v\&s las $4 \mathrm{C}$ del consumidor

Figure 1. $4 P^{\prime} s \vee \& s 4 C^{\prime} s$ consumer

Fuente: McClean, R. (2012). The $4 C^{\prime}$ 's versus the 4 P's of Marketing. Custom Fit. Recuperado de http://www.customfitonline.com/news/2012/10/19/4-cs-versusthe-4-ps-of-marketing/

\section{ESTRATEGIAS}

Una vez estudiado el contexto de Marketing mix, se presentan las estrategias de los autores Miles y Snow (1978) basadas en tres grandes hipótesis: la primera de ellas es que las organizaciones con éxito desarrollan a lo largo del tiempo una adaptación sistemática al entorno, con esta premisa, se destaca el ciclo adaptativo como la representación de una filosofía general de comportamiento organizativo; la segunda premisa es que existen cuatro orientaciones estratégicas dentro de cada industria; la tercera premisa de esta tipología, se refiere al resultado obtenido con cada estrategia. De ahí la importancia del marketing estratégico, el cual permite detectar las necesidades del mercado aprovechando oportunidades y utilizando los recursos disponibles de manera eficiente, para alcanzar objetivos y lograr ventajas competitivas (Molina, 2012).
Es por eso que el marketing estratégico es una herramienta fundamental para el posicionamiento de micro, pequeñas y medianas empresas, dado que las estrategias orientan las decisiones y permiten que los planes operativos se realicen de forma concurrente al logro de los objetivos de la empresa con la finalidad de incrementar las posibilidades de cumplimiento de objetivos y metas (Urdaneta, 2012; Aguilera, 2011).

Estrategia, es el arte de elegir y dosificar los medios disponibles y/o determinar las mejores líneas de actuación posibles (en un momento dado) para poder optimizar los objetos asignados (Ferré, 2002). Si una organización quiere tener la oportunidad de alcanzar sus metas y objetivos debe contar con un plan de juego o un mapa para llegar hasta ellos. En efecto, una estrategia resume el plan de juego para que una organización logre el éxito (Ferrell y Hartline, 2008). 
La capacidad de las empresas para adaptarse a los mercados es un tema central en la literatura sobre dirección estratégica (Grant, 1991). Así, la gestión estratégica se puede considerar como un proceso de adaptación continua a los cambios en el entorno interno y externo de la organización (Chakravarthy, 1992). El objetivo básico es adecuar los recursos internos de la empresa a las oportunidades surgidas en su entorno, desarrollar la orientación organizativa de la compañía, crear estructuras apropiadas y comunicar un propósito (Stalk, Evans y Schulman, 1992).

De acuerdo con el concepto de capacidad adaptativa, y en términos de los tres problemas arriba expuestos, las distintas formas organizativas pueden ser reducidas a varios arquetipos. Cada uno de ellos posee su propia estrategia para relacionarse con el(los) mercado(s) elegido(s) y representa una configuración particular de tecnología, estructura y procesos administrativos, coherentes con su estrategia de mercado (Snow, Miles y Coleman, 1992).

La dimensión clave que subyace en esta clasificación es la velocidad con que las organizaciones reaccionan ante cambios en el entorno, modificando sus productos y los mercados a los que se dirigen: las organizaciones defensivas son aquellas con dominios de producto mercado reducidos. Se caracterizan por ofrecer un conjunto relativamente estable de productos $\mathrm{y} / \mathrm{o}$ servicios a un segmento del mercado en el que se especializan. Tienden a ignorar los cambios que no les afectan directamente en sus dominios, y su orientación básica es mejorar la eficiencia de sus operaciones actuales. Estas organizaciones suelen adoptar una estructura funcional, tecnologías intensivas en capital y presentan competencias en producción, ingeniería de procesos y control de costes.

Las organizaciones exploradoras se sitúan en el extremo opuesto. Se trata de empresas que buscan continuamente nuevas oportunidades de mercado y que intentan ser las primeras en ofrecer nuevos productos o en desarrollar nuevos mercados. Así, con frecuencia son las creadoras del cambio y la incertidumbre a los que deben responder sus competidores. Su énfasis en la innovación de productos y mercados les impide ser totalmente eficientes. Se caracterizan por la diversificación de la línea de productos, el empleo de múltiples tecnologías, una estructura divisional y competencias en $I+D$, investigación de mercados e ingeniería de desarrollo (Miles y Snow, 1978).

Las organizaciones analizadoras poseen características propias de las defensivas y exploradoras. Es decir, mantienen un dominio de producto mercado relativamente estable mientras que, al mismo tiempo, buscan el desarrollo selectivo de nuevos productos $\mathrm{y} / \mathrm{o}$ mercados. En sus áreas estables, funcionan rutinaria y eficientemente mediante el uso de estructuras y procesos formalizados. En las áreas más dinámicas, observan atentamente las nuevas ideas de los competidores y luego adoptan con rapidez aquellas 6 que parecen más prometedoras. Se caracterizan por ofrecer una línea de productos básicos limitada, la selección de un pequeño número de productos relacionados, una estructura generalmente matricial, y dominan las competencias de producción, ingeniería de procesos y marketing. Las organizaciones reactivas son aquellas incapaces de responder de forma efectiva a los cambios e incertidumbres percibidos. Este tipo de organizaciones carece de una relación estrategiaestructura consistente, por lo que no realizan ajustes hasta que no son forzadas por las presiones del entorno (Miles y Snow, 1978).

De acuerdo con el análisis realizado, tanto del mix marketing como de las estrategias organizativas y adaptativas, se pude establecer que existe una relación directa entre estos dos factores, como se muestra a continuación en la Fig. 2. 

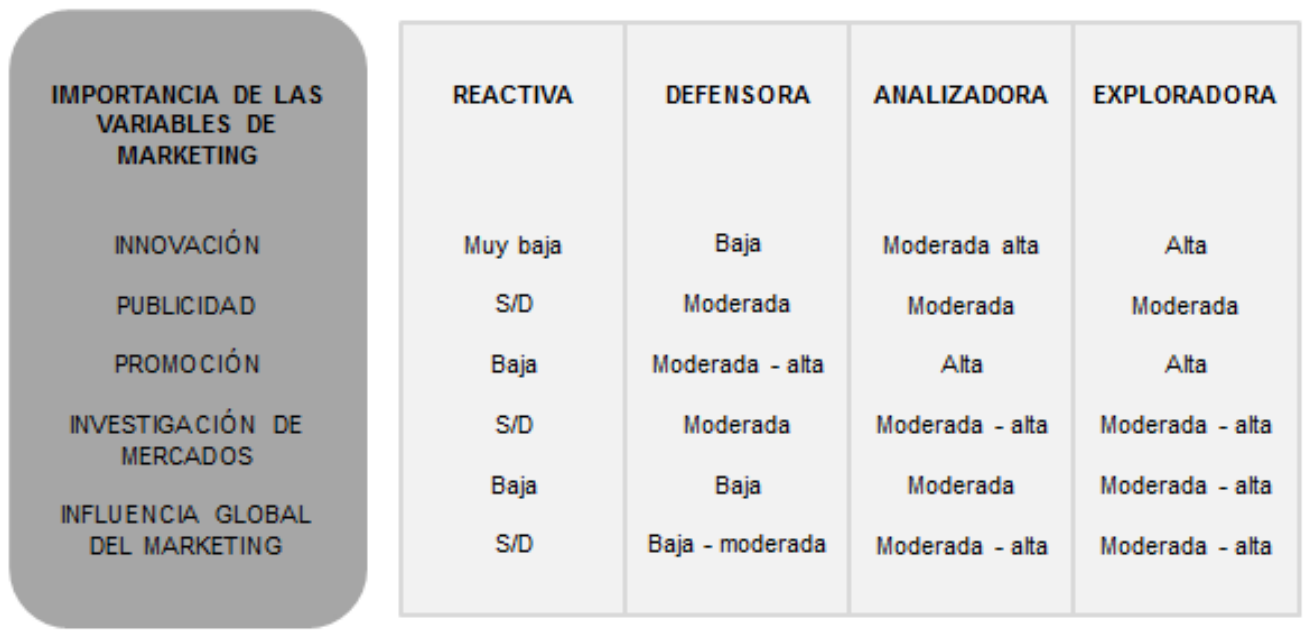

Figura 2. Relación directa el Marketing mix y las estrategias

Figure 2. Direct relationship between Marketing mix and strategies

Fuente: Munuera, J. L. y Rodríguez, A. I. (2012) Estrategias de marketing. 2 da. Edición. Editorial ESIC, Madrid: España.

\section{METODOLOGÍA}

La presente investigación tiene como objetivo conocer el impacto que tienen las estrategias en el Marketing mix de la Mipyme del barrio de La Purísima, en el municipio de Aguascalientes. Es una investigación cuantitativa, con un método descriptivo - correlacional, de corte transversal; el tamaño de muestra es de 133 micro, pequeñas y medianas empresas del sector comercial, con un nivel de confianza de $95 \%$ y $8 \%$ de margen de error, siendo esta una muestra aleatoria simple, con base al Directorio Estadístico Nacional de Unidades Económicas (DENUE, INEGI 2013). Los datos se recolectaron entre octubre y noviembre de 2013. Para la presente investigación, se realizó una encuesta que se componía de 51 ítems en total de las dos variables de estudio: Estrategias con 16 ítems y Marketing mix con 35 ítems, cada uno de ellos fue medido mediante la escala de Likert (1-5) tomando el valor de 1, como menor importancia y 5 como mayor importancia, el instrumento se aplicó a los propietarios de las empresas estudiadas; para este, se utilizó la técnica de regresión lineal con el fin de dar a conocer la viabilidad de modelo teórico de la investigación, que a continuación se muestra en la Fig. 3, para ello se plantean las siguientes hipótesis:

$\mathrm{H}_{1}$ : Las estrategias exploradoras impactan de manera positiva en el Marketing mix de las Mipymes en la zona de La Purísima del municipio de Aguascalientes, México.

$\mathrm{H}_{2}$ : Las estrategias analizadoras impactan de manera positiva en el Marketing mix de las Mipymes en la zona de La Purísima del municipio de Aguascalientes, México.

$\mathrm{H}_{3}$ : Las estrategias defensivas impactan de manera positiva en el Marketing mix de las Mipymes en la zona de La Purísima del municipio de Aguascalientes, México.

$\mathrm{H}_{4}$ : Las estrategias reactivas impactan de manera positiva en el Marketing mix de las Mipymes en la zona de La Purísima del municipio de Aguascalientes, México. 


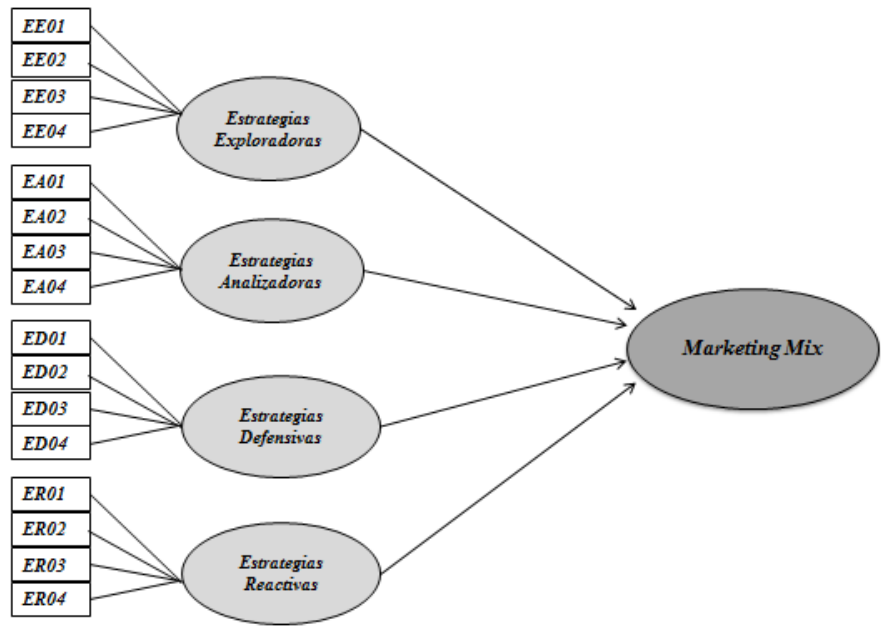

Figura 3. Modelo Teórico de la Investigación

Figure 3. Theoretical Model of Research

Fuente: Elaboración propia a partir de la adaptación de Rodríguez (2012) de Miles y Snow (1978) y Androta (2006).

\section{RESULTADOS}

Los resultados de la presente investigación fueron realizados a través del uso de la técnica estadística Regresión lineal, bajo el método de pasos sucesivos, a través del software estadístico SPSS V20, con el objetivo de probar las hipótesis de investigación. Para el mejor entendimiento de los resultados plasmados, es necesario comprender que la técnica estadística de Regresión lineal permite desarrollar un modelo para predecir los valores de una variable dependiente y una variable independiente para determinar el grado de influencia que existe entre las variables propuestas, como en (1)

$$
Y 0=\beta 0+\beta 1 X 1+z
$$

En donde:

$\mathrm{YO}=$ Variable dependiente

$\beta 0=$ Intersección o término constante

$\beta 1=$ Parámetros de medición de influencia de las variables independientes

$\mathrm{X} 1=$ Variable independiente

$z=$ Perturbación aleatoria que recoge todos aquellos factores de la realidad no controlables u observables.
La prueba de Durbin-Watson representa el valor de aceptación del modelo planteado; por otro lado, el FIV mide la colinealidad de la relación de la variable $X$ con la ecuación de la Regresión lineal. La significancia explica lo representativo del modelo de regresión estadísticamente con un nivel de confianza del 95\%. Por último, la ecuación (1) se compone de dos valores representativos, $\beta 0$ indica el valor de la constante o intersección y $\beta 1$ es el parámetro de medición de influencia de la variable independiente (Sheldon, 2007).

A continuación, en la Tabla 2 se muestra el primer resumen del modelo, en el que se obtuvo un valor de $R$ de 0.547 y un $R^{2}$ de 0.299 , lo que indica que la variable Estrategias exploradoras está correlacionada con un $54.7 \%$ con el constructo de Markentig mix de las Mipymes de la zona de La Purísima y la estructura del modelo teórico se explica un $29.90 \%$.

De la misma manera, se presenta en la Tabla 3 el segundo resumen del modelo, en él se obtuvo un valor de $R$ de 0.302 y una $R^{2}$ de 0.091 , lo que indica que las Estrategias analizadoras se encuentran con una correlación del 30.2\% con el Marketing mix de las Mipymes estudiadas, y el modelo teórico es explicado un 9.1\%. 
Tabla 2. Resumen del Modelo $1^{\mathrm{b}}$

Table 2. Summary of the first model ${ }^{b}$

$\begin{array}{lr}\text { Modelo } & 1 \\ R & 0.547^{a} \\ \mathrm{R}^{2} & 0.299 \\ \mathrm{R}^{2} \text { corregida } & 0.294 \\ \text { Error típico de la } & 0.65816 \\ \text { estimación } & 1.854\end{array}$

a. Variable predictora (Constante), Estrategias Exploradoras

b. Variable dependiente: Marketing mix

Fuente: Elaboración propia a partir de los datos obtenidos en el software SPSS versión 20.

Tabla 3. Resumen del segundo Modelob Table 3. Summary of the second model ${ }^{b}$

\begin{tabular}{|c|c|}
\hline \multicolumn{2}{|l|}{ Modelo } \\
\hline $\mathrm{R}$ & $0.302^{\mathrm{a}}$ \\
\hline $\mathrm{R}^{2}$ & 0.091 \\
\hline $\mathrm{R}^{2}$ corregida & 0.084 \\
\hline $\begin{array}{l}\text { Error típico de la } \\
\text { estimación }\end{array}$ & 0.74945 \\
\hline Durbin-Watson & 2.142 \\
\hline \multicolumn{2}{|c|}{$\begin{array}{l}\text { a. Variable predictora (Constante), } \\
\text { Estrategias Analizadoras } \\
\text { b. Variable dependiente: Marketing Mix } \\
\text { uente: Elaboración propia a partir de los datos } \\
\text { obtenidos en el software SPSS versión } 20 .\end{array}$} \\
\hline
\end{tabular}

Tabla 4. Resumen del tercer Modelob Table 4. Summary of the third Model ${ }^{b}$

\begin{tabular}{lr}
\hline Modelo & 3 \\
\hline$R$ & $0.518^{\mathrm{a}}$ \\
\hline $\mathrm{R}^{2}$ & 0.269 \\
\hline $\mathrm{R}^{2}$ corregida & 0.263 \\
\hline $\begin{array}{l}\text { Error típico de la } \\
\text { estimación }\end{array}$ & 0.67238 \\
\hline $\begin{array}{l}\text { Durbin-Watson } \\
\text { a. Variable predictora }\end{array}$ & (Constante), \\
$\begin{array}{l}\text { Defensivas } \\
\text { b. Variable dependiente: Marketing mix }\end{array}$ \\
$\begin{array}{l}\text { Fuente: Elaboración propia a partir de los datos } \\
\text { obtenidos en el software SPSS versión 20. }\end{array}$
\end{tabular}

Así mismo, se presenta en la Tabla 4, el tercer resumen del modelo referente a la variable
Estrategias defensivas con el Marketing mix, donde se obtuvo un 0.518 como valor de $\mathrm{R}$ y una $\mathrm{R}^{2}$ de 0.269 , lo que expresa que las Estrategias defensivas están correlacionadas en un $51.8 \%$ con el Marketing mix de las Mipymes de la zona de La Purísima, y el modelo teórico es un $26.9 \%$.

En la Tabla 5 se presenta el cuarto resumen del modelo, en el que se obtuvo un valor de $\mathrm{R}$ de 0.220 y una $R^{2}$ de 0.048 , lo que indica que la variable de Estrategias reactivas esta correlacionada en un $22 \%$ con la variable el Marketing mix de las pequeñas y medianas empresas estudiadas, y la estructura del modelo teórico se explica en un $4.8 \%$.

Tabla 5. Resumen del cuarto Modelo ${ }^{b}$ Table 5. Summary of the fourth Model ${ }^{b}$

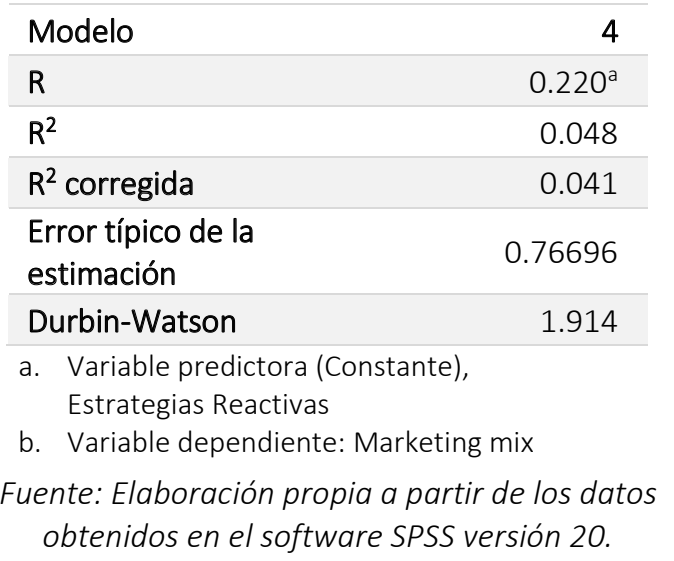

Sin embargo, de acuerdo con los resultados de la regresión lineal que se presentan en la Tabla 6 , se concluye que alrededor de un $54.7 \%$ del Marketing mix de las Mipymes de la Zona de La Purísima se debe las Estrategias exploradoras, al influir esta de manera significativa, con un valor de $\mathrm{t}$ de 6.606, a un nivel de significancia de 0.000; así mismo, las Estrategias analizadoras influyen en un $30.2 \%$ en el Marketing mix, ya que su valor de $t$ es de 10.936, a un nivel de significancia de 0.00; de la misma manera las Estrategias defensivas influyen de manera significativa en un $51.8 \%$ en el Marketing mix, con un valor de $t$ de 7.873, a un nivel de significancia de 0.000 y por último, las Estrategias reactivas influyen en un $22 \%$ en el Marketing mix, con un valor de $t$ de 14.247, a un nivel de significancia de 0.001 . 
Tabla 6. Resultado de Análisis de Regresión lineal Table 6. Result Linear Regression Analysis

\begin{tabular}{|c|c|c|}
\hline Variables & Competitividad & Resultados \\
\hline \multirow{3}{*}{$\begin{array}{l}\text { Estrategias } \\
\text { Exploradoras }\end{array}$} & \multirow[t]{2}{*}{$0.547^{* *}$} & $\mathrm{R}^{2}$ Ajustada $=0.294$ \\
\hline & & Valor de $F=55.914$ \\
\hline & $(6.606)$ & FIV más alto $=1.000$ \\
\hline \multirow{3}{*}{$\begin{array}{l}\text { Estrategias } \\
\text { Analizadoras }\end{array}$} & \multirow[t]{2}{*}{$0.302^{* *}$} & $\mathrm{R}^{2}$ Ajustada $=0.084$ \\
\hline & & Valor de $F=13.154$ \\
\hline & $(10.963)$ & FIV más alto= 1.000 \\
\hline \multirow{3}{*}{$\begin{array}{l}\text { Estrategias } \\
\text { Defensivas }\end{array}$} & \multirow[t]{2}{*}{$0.518^{* *}$} & $\mathrm{R}^{2}$ Ajustada $=.263$ \\
\hline & & Valor de $F=48.092$ \\
\hline & (7.873) & FIV más alto $=1.000$ \\
\hline \multirow{3}{*}{ Estrategias Reactivas } & \multirow[t]{2}{*}{$0.220^{*}$} & $\mathrm{R}^{2}$ Ajustada $=.041$ \\
\hline & & Valor de $F=6.646$ \\
\hline & $(14.247)$ & FIV más alto= 1.000 \\
\hline \multicolumn{3}{|l|}{$\begin{array}{l}* * * P<0.001 ; * * P<0.05 \\
\text { El valor entre paréntesis } r\end{array}$} \\
\hline
\end{tabular}

En cuanto a las hipótesis formuladas en la investigación, se puede concluir que de acuerdo con la $\mathrm{H}_{1}$, los resultados obtenidos $(\beta=0.547, p<$ $0.05)$ indican que las Estrategias exploradoras tienen efectos positivos y significativos en el Marketing mix de las Mipymes de la zona de La Purísima, por lo tanto al influir un $54.7 \%$, se acepta la $H_{1}$. En cuanto a la $H_{2}$ se obtuvo $(\beta=0.302$, $p<0.05)$, lo cual indica que las Estrategias analizadoras tienen efectos positivos en el Marketing mix, al influir esta variable un $30.2 \%$, se acepta la $\mathrm{H}_{2}$. Para la $\mathrm{H}_{3}$ es lo mismo, se acepta, debido a que se obtuvieron resultados significativos ( $\beta=0.518, p<0.05$ ), lo cual expresa que las Estrategias defensivas impactan de manera importante en el Marketing mix, al contar con una influencia de $51.8 \%$. Por último, para la $\mathrm{H}_{4}$, los resultados obtenidos $(\beta=0.220, \mathrm{p}<0.05)$, indican que las Estrategias reactivas inciden muy poco en el Marketing mix de las micro empresas de la zona estudiada, al influir esta variable un $22 \%$, sin embargo nuevamente se acepta la $\mathrm{H}_{4}$.

Como último proceso de los resultados, en la Tabla 7 se muestran las ecuaciones de la Regresión lineal, haciendo referencia al primer modelo, se presenta mediante el valor de Y1 que muestra la variable de Marketing mix de las Mipymes de la zona de La Purísima de Aguascalientes, lo que indica que de acuerdo con la Ecuación de Regresión, el Marketing mix está en función de las variables de Estrategias exploradoras (es una media de 3.8759), las Estrategias analizadoras (en una media de 3.1579), las Estrategias defensivas (en una media de 3.3459) y las Estrategias reactivas (en una media de 2.9361), utilizando 1 error estándar en las 4 dimensiones del modelo.

Tabla 7. Ecuaciones de la Regresión Lineal

Table 7. Linear Regression equations

Ecuación

$$
\begin{aligned}
& Y 1=\beta 0+\left(\beta 1^{*} \text { Estrategias Exploradoras }\right) \pm \mathrm{e} \\
& Y 1=\beta 0+\left(\beta 1^{*} \text { Estrategias Analizadoras }\right) \pm \mathrm{e} \\
& \mathrm{Y} 1=\beta 0+\left(\beta 1^{*} \text { Estrategias Defensivas }\right) \pm \mathrm{e} \\
& \mathrm{Y} 1=\beta 0+\left(\beta 1^{*} \text { Estrategias Reactivas }\right) \pm \mathrm{e}
\end{aligned}
$$

Resultados Marketing Mix $=1.461+(.412 * 3.8759) \pm 0.221$

Marketing Mix $=2.326+(.232 * 3.1579) \pm 0.212$

Marketing Mix $=1.656+(.419 * 3.3459) \pm 0.210$

Prácticas de Calidad $=2.617+(.150 * 2.9361) \pm 0.184$

Fuente: Elaboración propia a partir de los datos obtenidos en el software SPSS versión 20 


\section{CONCLUSIONES}

En los tiempos actuales estudiar una clasificación de empresas tan incierta, como lo son las Mipymes en México, se vuelve un gran reto debido a las circunstancias internas y externas que enfrentan: los mercados están cambiando continuamente, los avances tecnológicos se modifican con frecuencia, entre otros elementos que se vuelven difíciles de medir por parte de este segmento de empresas. Aguascalientes, en la actualidad, presenta un desarrollo industrial meramente manufacturero, lo cual incide de manera negativa en el crecimiento de las Mipymes del estado, ya que existen altas barreras de entrada a dicho sector. Sin amargo, estas empresas encuentran oportunidades en el sector comercial por su naturaleza, tal es el caso de la zona de La Purísima en el municipio de Aguascalientes.

Esta zona, a lo largo de la historia de Aguascalientes ha sido un parteaguas en aportar índices positivos al sector comercial, debido a que en sus inicios formaba parte de la zona comercial más recurrente por parte de los hidrocálidos. Sin embargo, las empresas que se lograron mantener y las de nueva creación han enfrentado una serie de cambios que han sido representados en sus ventas, esto se ha dado gracias al crecimiento del estado.

Como conclusión de esta investigación, las estrategias exploradoras y defensivas inciden de manera positiva en el Marketing mix, dicha incidencia positiva entre estas estrategias es porque los propietarios de estas empresas se encuentran en una continua búsqueda de nuevas oportunidades de negocios, a través del desarrollo solo de los productos que comercializan hacia un segmento muy concreto del mercado, sin embargo, no buscan nuevas oportunidades fuera de su negocio sino que su objetivo es la preservación de su cuota de mercado. Las principales innovaciones que suelen aplicar estas empresas se relacionan con el proceso productivo con el objetivo de incrementar su eficiencia.
Sin embargo, las estrategias analizadoras y reactivas son las que generaron menor impacto en el Marketing mix de las Mipymes estudiadas, es decir, estas empresas requieren hacer esfuerzos por mejorar o implementar aspectos de innovación en sus productos para atacar nuevos mercados; también es necesaria la implementación de campañas o elementos publicitarios que les permitan llegar a través de diferentes medios a los consumidores.

La fuerza de ventas es un elemento primordial, ya que les permitiría ampliar la posibilidad de venta en lugar de solo esperar a que el consumidor llegue al mostrador. A su vez, requieren implementar y generar mayores promociones de la gama de productos que venden. Todo esto debe ser a través de información confiable que se genera al realizar investigaciones de mercados, que les proporcione certeza a los empresarios sobre qué técnicas de la mezcla de marketing podrían funcionar.

\section{REFERENCIAS}

Aguilera Enríquez, L., González Adame, M. y Rodríguez Camacho, R. (2011). Estrategias empresariales para la competitividad y el crecimiento de las Pymes. Una evidencia empírica. Investigación y Ciencia, 19(53) 39$48 . \quad$ Recuperado de http://www.redalyc.org/articulo.oa?id=67421 408005.

Alcántara, R.; Cerón, A. y Goytortúa, C. (2012). Mercadotecnia para las MIPYMES: el reto de estrategias efectivas, innovadoras y a bajo costo. Memoria de la Quinta Cátedra CUMEX 2010 Segunda Sesión. Villahermosa, Tabasco, México. ISBN: 978-607 7557-79-1.

Aragón, A.; Rubio, A.; Serna, A. y Chablé, J. (2010). Estrategia y competitividad empresarial: Un estudio en las MiPyMEs de Tabasco. Aguascalientes, México.

Blech, G. y Blech, M. (2004). Publicidad y promoción de la comunicación de marketing integral. 6a Edición. México: Mc Graw Hill. 
Chakravarthy, B. S. (1992). Adaptation: a promising metaphor for strategic management. Academy of Management Review, 7(1), 35-44.

Cardozo, E. (2012). La definición de Pyme en América: una revisión del estado del arte. "6th International Conference on Industrial Engineering and Industrial Management y del XVI Congreso de Ingeniería de Organización". Vigo, España.

Dávila, A. y Rojo, M. (2010). La evaluación del rendimiento en empresas intensivas en conocimiento: un caso de estudio. Universia Business Review, 27, 124-141.

Ferrell, O. y Hartline, M. (2012). Estrategia de Marketing. 1a Edición. México: Cengage Learning.

Ferré, J. M. (2002). 101 Estrategias de negocios y de marketing. 1a Edición. España: Deusto.

Ferrell, O. C. y Hartline, M. (2008). Estrategia de Marketing. 3a Edición. México: D. F.: Cengage Learning.

Grant, R. M. (1991). The resource-based theory of competitive advantage: Implications for strategy formulation. California Management Review, primavera, 1(3), 114.

González, G. y Bermúdez, T. (2010). Fuentes de información, indicadores y herramientas más usadas por gerentes de Mipyme en Cali, Colombia. Contaduría y Administración, 232, 83-108.

González Valiente, C. L. (2014). El profesional de la información como gestor de mercadotecnia: aproximación a un importante rol. Information Science \&Library Science, 24(2) 10 . Recuperado de http://www.researchgate.net/profile/Carlos_ Luis_Gonzalez-Valiente?ev=hdr_xprf

Islas, A. (2010). La mercadotecnia: una estrategia de competitividad. $\mathrm{V}$ Encuentro Estatal de Investigación en Ciencias Económico Administrativas, Pachuca, Hgo. ISBN: 978607-482-132-1.
Kotler, P. y Armstrong, G. (2004) Marketing. 10a Edición: Pearson, Madrid.

McClean, R. (2012). The 4 C's versus the 4 P's of Marketing. Custom Fit. Recuperado de http://www.customfitonline.com/news/2012/ 10/19/4-cs versusthe-4-ps-of- arketing/

Miles, R. E. y Snow, C. C. (1978). Organizational strategy, structure and process. Ed. New York: McGraw Hill.

Montaño, F. (2005). Mercadotecnia. Investigación y análisis para el éxito. México, D.F.: Trillas.

Molina, L. (2012). Elaboración de un plan de marketing para el comisariato de la FAE de la ciudad de Latacunga, provincia de Cotopaxi período 2010 2012. Unidad Académica de Ciencias Administrativas y Humanísticas. UTC. Latacunga. 90.

Munuera, J. L. y Rodríguez, A. I. (2012). Estrategias de Marketing. 2da. Edición. Madrid: Editorial ESIC.

Rubio, A. y Aragón, A. (2009). SMEs competitive behaviour: strategic resources and strategies. Management Research, 7(3), 171-190.

Sheldon, M. R. (2007). Introducción a la estadística. Editorial Reverté. 1ạ Edición Barcelona: España.

Stalk, G.; Evans, P. y Schulman, L. (1992) Competing on capabilities: the new rules of corporate strategy. Harvard Business Review, 70(2), 57- 69 .

Snow, C. C.; Miles, R. y Coleman, H. (1992). Managing 21st century network organizations. Organizational Dynamics, 20(3), 5-20.

Urdaneta, M. (2012). Marketing estratégico para el posicionamiento de empresas de consultoría gerencial. 1, 594-600. Recuperado de http://virtual.uribe.edu/eventostexto/JN2/UR B-054.pdf. 
Uribe, G. (2010). El marketing como factor estratégico para la competitividad en las pymes manufactureras de Guadalajara, (1), 1$19 . \quad$ Recuperado de http://riico.org/memoria/quinto/RIICO3301.PDF.
Zevallos V. (2003). Micro, pequeñas y medianas empresas en América Latina. Revista de la CEPAL, 3(2), 1. 